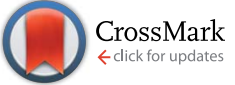

Cite this: RSC Adv., 2016, 6, 17023

\title{
The importance of raw graphite size to the capacitive properties of graphene oxide $\uparrow$
}

\author{
Haoran $\mathrm{Yu}{ }^{a}$ Keyu Xie, ${ }^{\text {*a }}$ Jingzhi Hu, ${ }^{a}$ Chao Shen, ${ }^{a}$ Jian-gan Wang ${ }^{a}$ \\ and Bingqing Wei ${ }^{\star a b}$
}

Carbon-based materials have been widely used in energy storage and conversion devices. Among them, graphene oxide (GO) holds much promise for supercapacitor applications due to its high-capacitance, low-cost, etc. Nevertheless, intrinsic characteristics related to the capacitive properties of GO are far from being fully understood. Little attention has been paid to the size effect of raw graphite on the electrochemical characteristics of GO. In order to investigate the relationship between raw graphite size and the capacitive properties of GO, electrochemical performances of GO, prepared from raw graphite of three different sizes using a modified Hummer's method, were compared. Experimental results indicated that the capacitance of a GO electrode was enhanced with the increasing size of the raw graphite. The GO electrode prepared with the largest size of raw graphite achieved a specific capacitance as high as $94.4 \mathrm{~F} \mathrm{~g}^{-1}$ at $0.1 \mathrm{~A} \mathrm{~g}^{-1}$ due to a medium-sized specific surface area and smaller charge transfer resistance. It is concluded that more attention should be paid to the prevention of agglomeration or even restacking of the graphene-based material after the heat drying of graphenebased solutions because of residual intercalated water molecules, which contribute to strong hydrogen bonding interactions between layers.

Received 19th November 2015 Accepted 27th January 2016

DOI: $10.1039 / \mathrm{c} 5 \mathrm{ra24501j}$

www.rsc.org/advances mechanical exfoliation of graphite flakes is a serendipitous method that only contributes to uneven films and is not suitable for large-scale production; epitaxial growth is a hightemperature process that is beyond the control of morphology and adsorption energy; the reduction of GO is a promising approach but the reduction to graphene is only partial with a fragile stability of the colloidal dispersion. ${ }^{11-13}$ Therefore, graphene still needs to compete with activated carbons in terms of cost and performance in order to be utilized in actual production. Nonetheless, the intermediate GO bears an interesting potential as a supercapacitor electrode material owing to its low cost and high capacitance. During the formation of GO, the conjugated structure is disrupted and the $\pi$-electrons are localized by the embedding of covalent functional groups. Afterwards, the covalent functional groups enhance the total capacitance through pseudo-capacitance. Also, they are functional sites and offer more opportunities for a secondary modification, such as doping with nitrogen or phosphorus. ${ }^{14}$ Taking its lower cost and high capacitance into account, GO may be a better choice than graphene as an electrode material for supercapacitors. ${ }^{15} \mathrm{~A}$ capacitance of up to $159 \mathrm{~F} \mathrm{~g}^{-1}$ in $\mathrm{H}_{2} \mathrm{SO}_{4}$ and $82 \mathrm{~F} \mathrm{~g}^{-1}$ in $\left(\mathrm{C}_{2} \mathrm{H}_{5}\right)_{4} \mathrm{NBF}_{4} /$ acetonitrile was reported for $\mathrm{GO},{ }^{10}$ and it showed a much higher volumetric capacitance than the majority of other carbon materials, which is a key factor for the development of energy storage devices. A higher capacitance of up to $189 \mathrm{~F} \mathrm{~g}^{-1}$ was obtained with GO electrodes in a symmetrical two-electrode cell. ${ }^{15}$ However, little attention has been
${ }^{a}$ State Key Laboratory of Solidification Processing and Center for Nano Energy Materials, Northwest Polytechnical University, Xi'an 710072, China. E-mail: kyxie@ nwpu.edu.cn

${ }^{b}$ Department of Mechanical Engineering, University of Delaware, Newark, DE19716, USA. E-mail: weib@udel.edu; Fax: +1-302-831-3619; Tel: +1-302-831-6438

$\dagger$ Electronic supplementary information (ESI) available. See DOI: $10.1039 / \mathrm{c} 5 \mathrm{ra} 24501 \mathrm{j}$ 
focused on the size effect of raw graphite on the electrochemical characteristics of GO for supercapacitors. It would be valuable to study the relationship between raw material size and the electrochemical performances of GO.

Herein, this paper mainly addresses a comparison of the electrochemical performances of GO prepared using a modified Hummers method from three different raw graphite sizes. It was found that the capacitance of the GO electrode is enhanced with the increasing size of the raw graphite. In addition, how to prevent GO from agglomerating or even restacking is also discussed. Our work will provide practical guidance to the synthesis of GO-based electrode materials for supercapacitors.

\section{Experimental}

\section{Synthesis of GO}

GO was prepared using a well-known modified Hummer's method, which has been described in detail elsewhere. ${ }^{16,17}$ The raw materials were natural graphite particles with sizes of 80 120 mesh, 750-850 mesh, and 2000 mesh (Aladdin), and the corresponding samples were designated as GO (100 mesh), GO (800 mesh), and GO (2000 mesh), respectively. Briefly, these raw graphite particles $(0.5 \mathrm{~g})$ were preoxidized with sulfuric acid (25 $\mathrm{ml}$ ) for $30 \mathrm{~min}$ at room temperature. Then, sodium nitrate $(0.5$ $\mathrm{g}$ ) and potassium permanganate ( $3 \mathrm{~g}$ ) were slowly dispersed into the mixture, which was immediately transferred into an ice bath with a temperature below $20{ }^{\circ} \mathrm{C}$ for $2 \mathrm{~h}$. Next, this mixture was stirred when the temperature was increased to $35{ }^{\circ} \mathrm{C}$. After oxidizing for $3 \mathrm{~h}$, the obtained mixture was diluted with $140 \mathrm{ml}$ of deionized water and stirred for another $2 \mathrm{~h}$ while being heated to a temperature of $95^{\circ} \mathrm{C}$. Then, hydrogen peroxide was poured into the mixture until the color of the mixture changed from brown to light yellow with no air bubbles being generated.. Afterwards, the obtained solution was washed with deionized water until the $\mathrm{pH}$ value became $6-7$ to obtain GO. The GO suspension was sonicated for $30 \mathrm{~min}$ at a power of about $200 \mathrm{~W}$. After a short rest for about $10 \mathrm{~min}$, the suspension was ultrasonicated for $10 \mathrm{~min}$ (UP400S, 0.4 cycle, 80\% intensity). The procedure above was repeated three times and the GO dispersion was obtained after centrifugation. Finally, the GO dispersion was dried at $80{ }^{\circ} \mathrm{C}$ in an air dry oven for about $24 \mathrm{~h}$ to get GO.

\section{Characterization techniques}

The GO morphology was characterized using scanning electron microscopy (SEM, JSM6700E) and transmission electron microscopy (TEM, JEOL JEM-3010F). Raman spectra were obtained using a HORIBA JOBIN YVON HR 800 and a Renishaw inVia instrument. X-ray photoelectron spectroscopy (XPS) was performed on an ESCALAB250xi XPS system. XRD data were measured using an X-ray diffractometer (D/max-2400, Rigaku Tokyo, Japan). Nitrogen adsorption-desorption isotherms of the samples were determined using a Surface Area and Porosity Analyzer (ASAP 2020 HD88). Square resistances were tested using a four-point probe meter (SZT-2A).

\section{Electrochemical measurements}

The electrochemical performances were tested on a workstation (Solartron analytical $1400+1470 \mathrm{E}$ ) using a three-electrode system at room temperature. Consequently, nickel foam (15 $\mathrm{mm} \times 15 \mathrm{~mm}$ ) dipped into the active material slurry was selected as the working electrode. The active material (about 4 mg per electrode after drying) contained GO, carbon black and a polyvinylidene fluoride (PVDF) binder at a mass ratio of $7: 2: 1$ dissolved in $N$-methyl-2-pyrrolidone (NMP). The electrode was dried at $60^{\circ} \mathrm{C}$ in a vacuum oven for $24 \mathrm{~h}$. Finally, the nickel foam coated with the active materials as the working electrode, a platinum foil as the counter electrode, a saturated calomel electrode as the reference electrode, and $6 \mathrm{M}$ potassium hydroxide solution as electrolyte were used. Cyclic voltammetry (CV) was performed with a potential window from $-0.8 \mathrm{~V}$ to $0 \mathrm{~V}$ at different scan rates $\left(5,10,20,30\right.$, and $\left.50 \mathrm{mV} \mathrm{s}^{-1}\right)$. Galvanic cycling (GC) was carried out with a voltage from $-0.7 \mathrm{~V}$ to $0 \mathrm{~V}$ at various current densities $\left(0.1,0.2,0.5,1\right.$, and $\left.2 \mathrm{~A} \mathrm{~g}^{-1}\right)$. In addition, the cyclic stability of the GO electrodes at a current density of $1 \mathrm{~A} \mathrm{~g}^{-1}$ for 1500 cycles was measured using GC. Electrochemical Impedance Spectroscopy (EIS) measurements were performed over a frequency range of $0.01 \mathrm{~Hz}$ to $10 \mathrm{kHz}$.

\section{Results and discussion}

\section{Morphological and structural characterizations}

The shape, size and intrinsic structure of raw graphite powders are characterized using SEM, these images of graphite powders with 2000 mesh, 850-750 mesh and 120-80 mesh are presented in Fig. 1(a-c). In Fig. 1(a), the particles have a wide particle distribution, but basically the particle size is less than $20 \mu \mathrm{m}$. As indicated in Fig. 1(b), the particle size is about 20 to $40 \mu \mathrm{m}$. Fig. 1(c) shows a relatively integrated slice of graphite with a size of more than $100 \mu \mathrm{m}$. Fig. 1(d) shows a TEM image of GO (2000 mesh) prepared using the modified Hummer's method. It
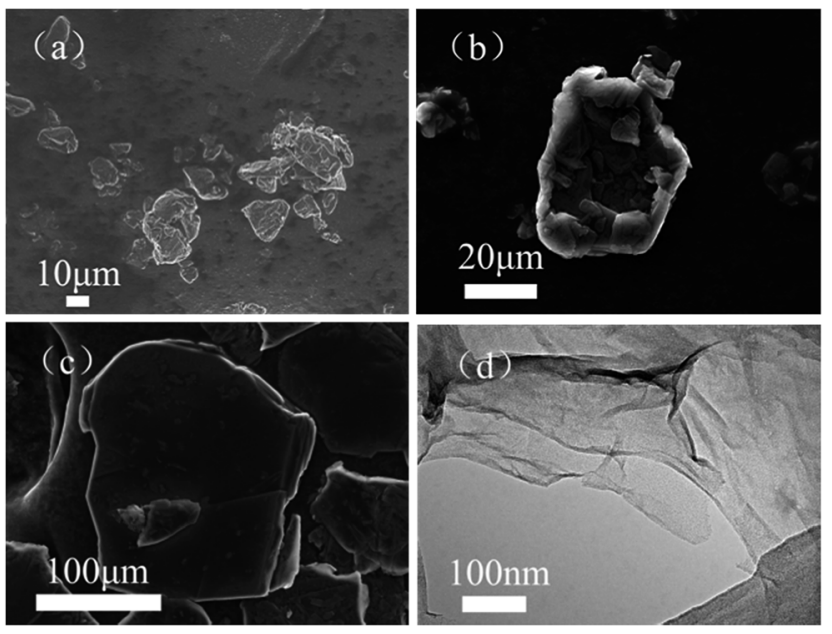

Fig. 1 SEM images of the raw graphite. (a) 2000 mesh graphite, (b) 850-750 mesh graphite and (c) 120-80 mesh graphite. (d) TEM image of GO (2000 mesh). 
demonstrates clearly that the prepared GO shows a silk veilwaves morphology with thin nanosheets.

It is well known that the $\mathrm{G}$ band of a Raman spectrum is a typical representation of the $E_{2 g}$ vibrational modes of the aromatic domains, whereas the $\mathrm{D}$ band is generated from the breathing modes of the graphitic domains. ${ }^{18-21}$ As shown in Fig. 2, obvious D and G bands are located at $1354 \mathrm{~cm}^{-1}$ and 1591 $\mathrm{cm}^{-1}$, respectively, the latter of which shifts a bit from the graphite Raman spectrum peak $\left(1580 \mathrm{~cm}^{-1}\right)$ after chemical oxidation. In addition, the intensity ratio of the $\mathrm{D}$ band to the $\mathrm{G}$ band $\left(I_{\mathrm{D}} / I_{\mathrm{G}}\right)$ is used to estimate the degree of disorder in graphene. ${ }^{18}$ Compared to that of raw graphite, the $I_{\mathrm{D}} / I_{\mathrm{G}}$ of all of the GOs obtained from different graphite sizes was increased dramatically, indicating high disorder in the GOs, arising from the addition of oxygen-containing functional groups. ${ }^{22}$ However, the sizes of the raw graphite have almost no effect on the Raman spectra, showing that the three samples have a similar graphitic degree.

XPS characterizations are further employed to analyze the elemental compositions and the configurations of the oxygencontaining functional groups in GO. The binding energy of $\mathrm{C}-\mathrm{C}$ is located at around $284.5 \mathrm{eV}$, and therefore the peaks of the oxygen-containing functional groups added to the graphite have a shift with respect to the $\mathrm{C}-\mathrm{C}$ bond. In Fig. 3(a), the $\mathrm{C} 1 \mathrm{~s}$ spectrum of the GO (2000 mesh) can be fitted into five peaks centered at 284.5, 285.2, 286.7, 287.2, and $288.6 \mathrm{eV}$, which could be assigned to the non-oxygenated ring $\mathrm{C}$ atoms $\mathrm{sp}^{3} \mathrm{C}(\mathrm{C}-\mathrm{C})$ and $\mathrm{sp}^{2} \mathrm{C}(\mathrm{C}=\mathrm{C})$, the $\mathrm{C}$ atoms in the epoxy/ether groups $(\mathrm{C}-\mathrm{O})$, the carbonyl $\mathrm{C}$ structure $(\mathrm{C}=\mathrm{O})$, and the carboxyl $\mathrm{C}$ structure $(\mathrm{O}-$ $\mathrm{C}=\mathrm{O}$ ), respectively. ${ }^{23}$ It can be clearly seen that the XPS spectra of GO (800 mesh) (Fig. 3(b)) and GO (100 mesh) (Fig. 3(c)) are similar to that of GO (2000 mesh). Meanwhile, the molar ratios of C/O for GO (2000 mesh), GO (800 mesh), and GO (100 mesh) are obtained as $2.2,2.1$, and 2.2, respectively, indicating that the oxidation of the three different raw materials is at almost the same level.

Fig. 4 shows XRD patterns of raw graphite, GO (2000 mesh), GO (800 mesh), and GO (100 mesh). In the XRD pattern of the raw graphite, there appears a (002) basal reflection with a sharp peak at $26.5^{\circ}$, and in the patterns for GO (2000 mesh), GO (800

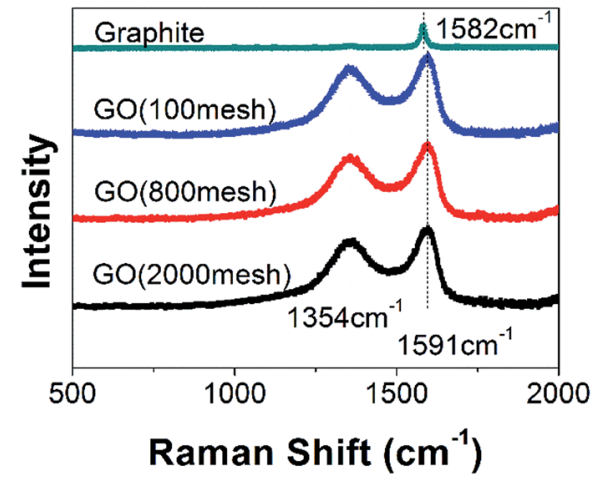

Fig. 2 Raman spectra of GO (2000 mesh), GO (800 mesh), GO (100 mesh) and graphite.
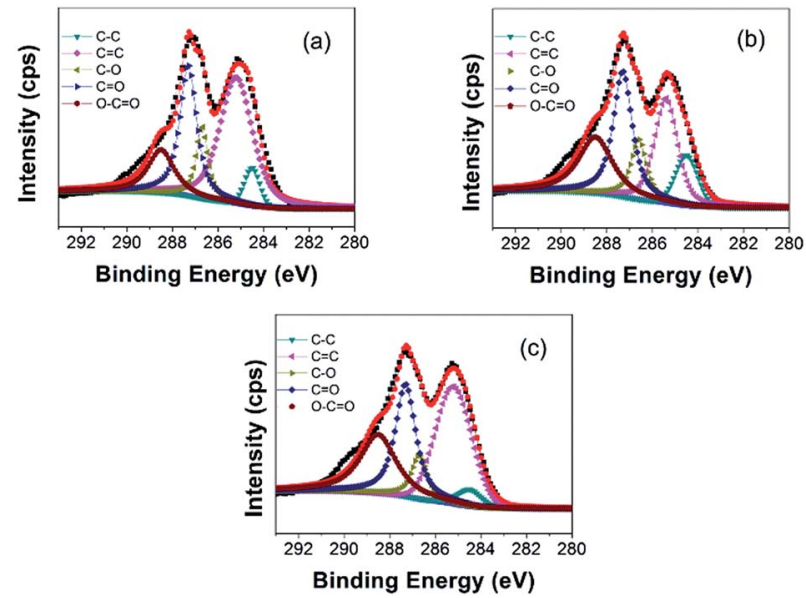

Fig. 3 XPS spectra of C1s in (a) GO (2000 mesh), (b) GO (800 mesh), and (c) GO (100 mesh).

mesh), and GO (100 mesh), broad peaks corresponding to (001) diffraction are found at $2 \theta=11.3^{\circ}, 11.2^{\circ}$ and $10.9^{\circ}$, respectively. Using the following Bragg equation:

$$
2 d \sin \theta=n \lambda
$$

where $d$ is the interlayer spacing of GO, $\theta$ is the diffraction angle, $n$ is the number of interlayers, and $\lambda$ is the X-ray wavelength of the $\mathrm{Cu}$ target, the interlayer spacings of GO (2000 mesh), GO (800 mesh) and GO (100 mesh) can be easily calculated as $0.782 \mathrm{~nm}, 0.789 \mathrm{~nm}$ and $0.811 \mathrm{~nm}$, respectively. According to the results from the Raman and XPS, theoxidation degrees of the three samples are similar. However, it is interesting to notice that the interlayer spacing increases with the increasing size of the particles. The appearance of this phenomenon may be caused by hydrogen bonding interactions. The interesting thing is that the "intercalated" water molecules remain stuck in the GO particles via hydrogen bonding interactions which facilitates interactions between the GO sheets, resulting in spontaneous agglomeration and even restacking to form graphite oxide after the GO solution has dried.,.$^{\mathbf{2 4 - 2 6}}$

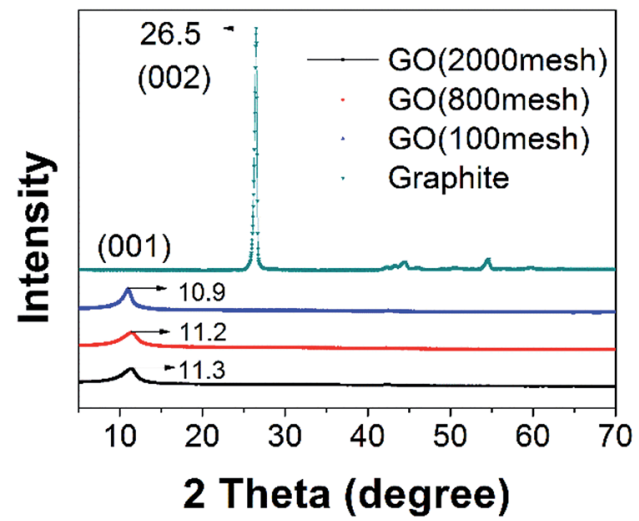

Fig. 4 XRD patterns of GO (2000 mesh), GO (800 mesh), GO (100 mesh) and graphite. 
Moreover, differently sized particles can cause different degrees of oxidation (in the carbon atoms between layers not from edges), which has an influence on the interlayer spacing between GO layers.

The hypothesis mentioned above is exactly substantiated by previous work. ${ }^{23,27}$ The formation of pristine GO occurs via the diffusion of an oxidizing agent into the graphite interlayer gallery, followed by a chemical reaction. Above all, this is the rate-determining step, and is diffusion-controlled by the oxidant replacing the acid intercalant. ${ }^{27}$ Consequently, graphite with a small size is oxidized significantly faster than large graphite, corresponding to there being more oxygen-containing functional groups between the layers under the same degree of oxidation, which contributes to strong hydrogen bonding interactions. Hence, hydrogen bonding interactions lead to the different interlayer spacing of the three samples, after the heat drying of GO solution, which coincides well with the XRD patterns. It is apparent that, in the preparation of GO, the GO would spontaneously agglomerate if there were no protective measures when heat drying GO solution.

Nitrogen adsorption-desorption isotherms at $77 \mathrm{~K}$ and pore size distributions of the three samples are shown in Fig. 5. It can be observed that all three samples belong to the type-I species according to IUPAC classification. ${ }^{28-30}$ The type-I isotherm evidently levels off below a relative pressure of 0.1 indicating that the GO samples are exclusively microporous. Moreover, the pore size distribution curves based on nonlinear density functional theory (NLDFT) confirm that the majority of these pores for the three GO samples were found to be less than $2 \mathrm{~nm}$ in diameter. As shown in Fig. 5, GO (2000 mesh), GO (800 mesh) and GO (100 mesh) have an undeveloped porosity with a specific surface area of 32,89 and $62 \mathrm{~m}^{2} \mathrm{~g}^{-1}$, respectively, which is contributed to by the defects and structure of GO. The data obtained here are a little lower than previous results. ${ }^{15}$ This result may be attributed to the agglomeration, or even the restacking of GO destroying the pore structure and thus reducing the specific surface area. As for smaller particles, GO (2000 mesh) originally tended to possess a larger surface area,

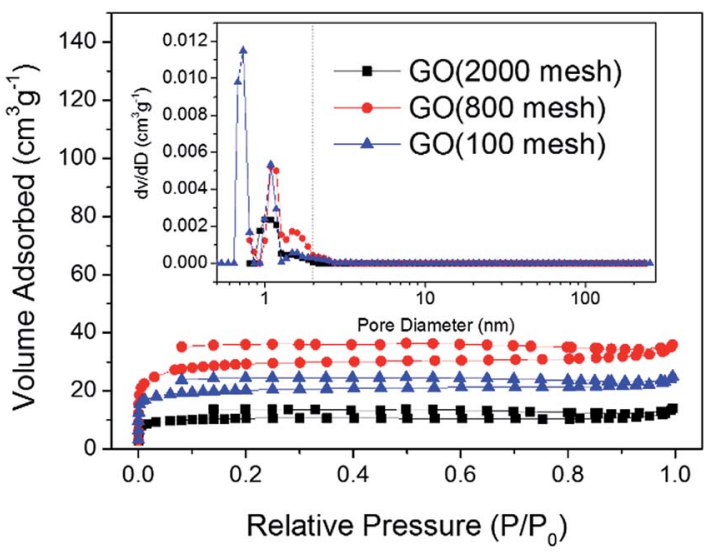

Fig. 5 Nitrogen adsorption-desorption isotherms and pore size distribution curves (inset) for GO (2000 mesh), GO (800 mesh), and GO (100 mesh). however, it contains more oxygen functional groups so that stronger hydrogen bonding interactions led to greater damage to the structure. This could be the reason that the specific surface area of GO (2000 mesh) is lower than that of GO (800 mesh). Evidently, GO suffers from severe restacking between individual sheets and thus loses its material identity and advantages. Therefore, the most important and challenging hurdle to achieve a high capacitance is to hinder the restacking of GO sheets. One of the approaches to solving this problem is the use of nonpolar aprotic "solvent", which doesn't interact with the oxygen groups of GO, thereby leading to the formation of crumpled non-stacked GO. For example, hydrophobic hexane as an anti-solvent was introduced to fabricate highly crumpled non-stacked GO nanosheets. By using hexane, it could be easily seen that the diffraction peak of GO was shifted from $11.38^{\circ}$ to $10.36^{\circ} .{ }^{24}$ Another solution to eliminate hydrogen bonding is via a condensation reaction of the hydroxyl end groups of polymer monomers with the hydroxyl or carboxylic acid groups on the GO sheets. For instance, GO was functionalized in a solution state with melamine resin monomers and the hydrogen bonding interactions were successfully prevented. ${ }^{26}$ In fact, rapid freeze-drying may be useful to remove water molecules, because the fluffy porous structure could be maintained by freezing in less than 5 seconds. For example the specific area of GO (100 mesh) can be improved to $158 \mathrm{~m}^{2} \mathrm{~g}^{-1}$ using rapid freeze-drying (ESI Fig. S1†).

\section{Electrochemical analysis}

Cyclic voltammogram analysis is a universal method to evaluate capacitive behavior. As is known, GO has low conductivity. The square resistances of the GO samples are about $120 \mathrm{MOhm}$ $\mathrm{sq}^{-1}$, measured using a four-point probe meter. Therefore a small quantity of carbon black was added to the electrode and nickel foam was used as a current collector. The CV curves of nickel foam and the three GO electrodes at $50 \mathrm{mV} \mathrm{s}^{-1}$ are shown in Fig. 6(a). Obviously, the current collector, nickel foam, has a negligible capacitance. The integrated areas for the GO
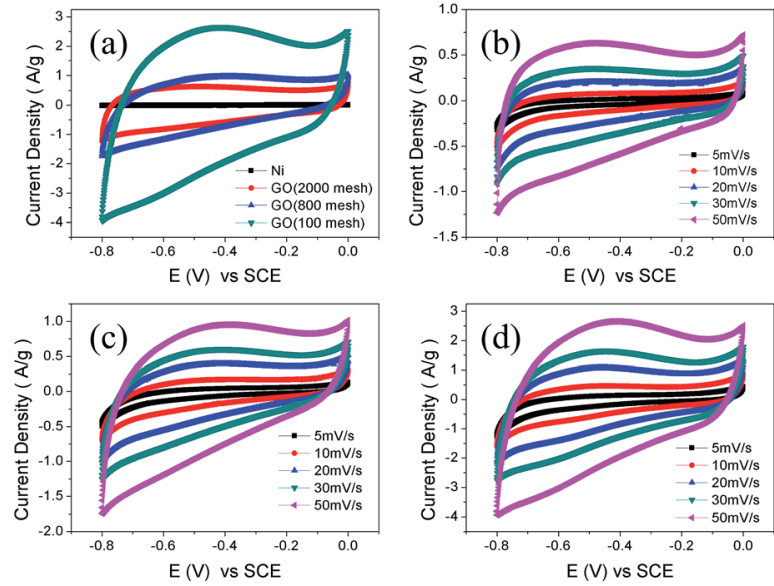

Fig. 6 (a) Cyclic voltammograms of different electrode materials at 50 $\mathrm{mV} \mathrm{s}^{-1}$. Cyclic voltammograms at different voltage scanning rates of (b) GO (2000 mesh), (c) GO (800 mesh), and (d) GO (100 mesh). 
electrodes intuitively grow with the increase of the raw graphite size, which is an indication of superior capacity and rapid diffusion of electrolyte ions from the solution into the pores of the electrodes made from larger raw materials. As shown in Fig. 6(b)-(d), the CV curves of GO (2000 mesh), GO (800 mesh), and GO (100 mesh) measured at low scan rates, exhibit an almost rectangular shape, while the CV curves become a little distorted at a high scan rate. Xu et al. investigated the rate capability of GO electrodes and demonstrated that the oxygencontaining functional groups could participate in quick pseudo-faradaic charge-discharge reactions. That means that GO electrodes have a quick charge propagation capability of both double layer capacitance and pseudo-capacitance. ${ }^{15}$

To obtain a more accurate specific capacitance of the GO electrodes for supercapacitors, galvanic cycle tests were further performed according to eqn (2):

$$
C=(I \Delta t) /(m \Delta U)
$$

where $I$ is the applied current (A), $\Delta t$ is the discharge time (s), $\Delta U$ is the potential range, and $m$ is the mass of active materials. Fig. 7(a)-(c) exhibit charge-discharge cycle curves of the GO electrodes at different current densities. The GO (100 mesh), GO (800 mesh) and GO (2000 mesh) electrodes deliver an initial specific capacitance of 94.4, 33.1 and $11.7 \mathrm{~F} \mathrm{~g}^{-1}$, respectively, at a current density of $0.1 \mathrm{~A} \mathrm{~g}^{-1}$. Fig. 7 (d) shows the specific capacitance as a function of current density in $6 \mathrm{M} \mathrm{KOH}$ for all samples. A decrease in specific capacitance was observed at high current densities owing to the slow diffusion of the electrolyte ions into the pores of the GO electrodes during the fast charge/discharge process. ${ }^{31,32}$

Typical Nyquist plots of the GO electrodes are shown in Fig. 8. The plots consist of a small semicircle at the high frequency region, which reflects the charge transfer process, and the inclined line in the low frequency range, representing the Warburg impedance, which shows the quick adsorption of
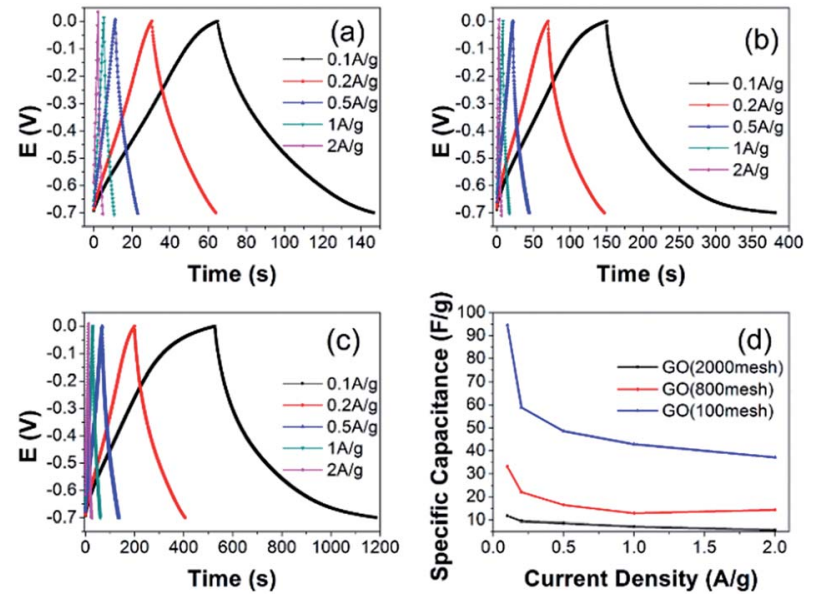

Fig. 7 Galvanic cycle curves for the GO electrodes on nickel foam: (a) GO (2000 mesh), (b) GO (800 mesh), and (c) GO (100 mesh). (d) Specific capacitance as a function of current density in $6 \mathrm{M} \mathrm{KOH}$ for all samples.

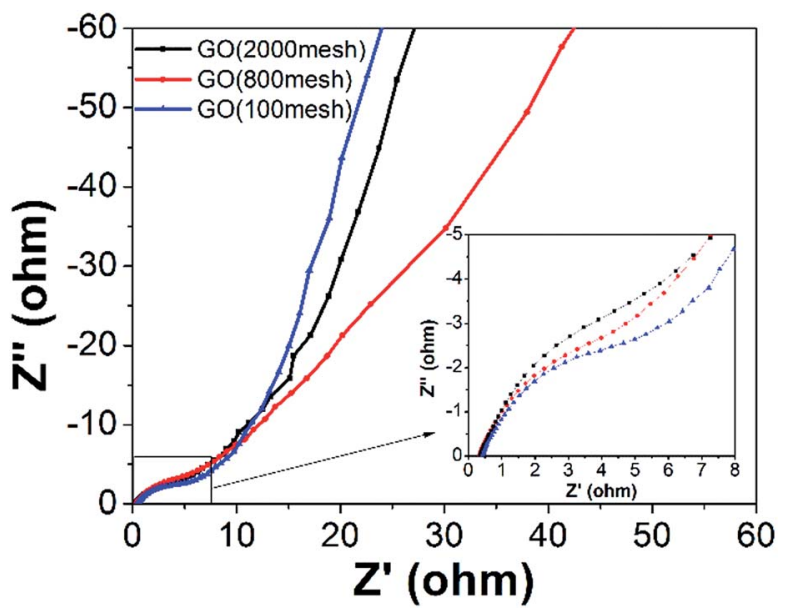

Fig. 8 The Nyquist plots of the three GO electrodes (the inset shows the enlarged EIS in the high frequency region).

ions onto the electrode surface and fast diffusion of ions. The observed equivalent series resistance (ESR) of GO (2000 mesh), GO (800 mesh), and GO (100 mesh) are similar and their charge transfer resistances decrease with the increase in raw graphite size. What's more, GO (100 mesh) has the fastest diffusion of ions while GO (800 mesh) has the slowest, which may be ascribed to different porous structure. Based on the above experimental results, GO (800 mesh) possesses the largest specific surface area, yet the corresponding electrode does not have the highest specific capacitance, among the GO samples prepared from the three different sizes of raw graphite. The reason for this is that the electrode containing GO (800 mesh) has a larger charge transfer resistance and the lowest diffusion of ions. Hence, it could be concluded that the capacitive properties of GO-based electrodes for supercapacitors are highly dependent on pore structure, specific surface area and electrical conductance properties.

Cycling lifetime is another important requirement to judge the electrochemical performances of electrodes. As shown in

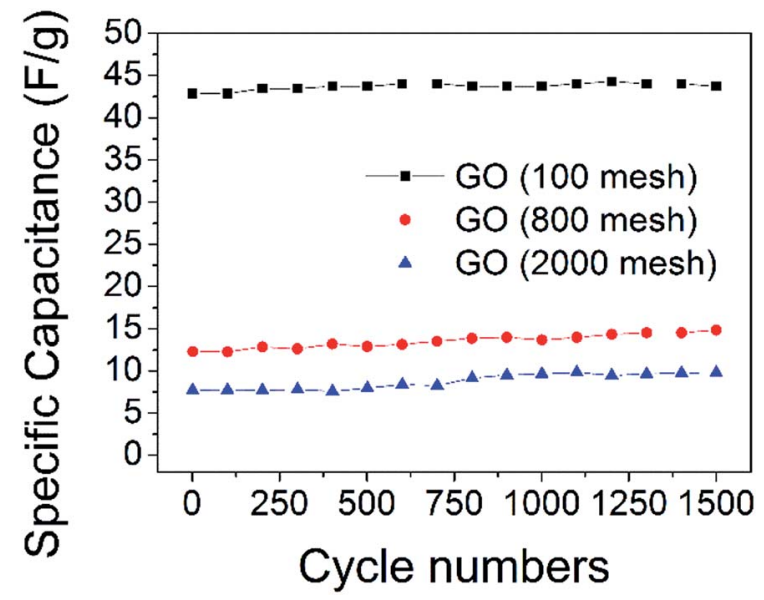

Fig. 9 Specific capacitance of the GO electrodes for 1500 cycles, charge-discharge tests at a current density of $1 \mathrm{~A} \mathrm{~g}^{-1}$. 
Fig. 9, there is almost no attenuation in the specific capacitance of all three GO electrodes after 1500 cycles, indicating that all of the GO samples have outstanding cycling stability. The XPS spectra of C1s in GO (100 mesh) after 1500 cycles show that some oxygen-containing functional groups were removed (ESI Fig. S2 $\uparrow$ ), and the molar ratios of $\mathrm{C} / \mathrm{O}$ of it increased to 2.7 from 2.2 , which means that at least $82 \%$ of the oxygen-containing functional groups are stable, which maintains high capacitance.

\section{Conclusions}

To sum up, a comparison of the electrochemical performances of GO electrodes from three different raw graphite sizes was systematically performed. The specific capacitance of the electrodes was $94.4,33.1$ and $11.7 \mathrm{~F} \mathrm{~g}^{-1}$ for GO (100 mesh), GO (800 mesh) and GO (2000 mesh), respectively, at a current density of $0.1 \mathrm{~A} \mathrm{~g}^{-1}$. The capacitance of the GO electrodes is enhanced with the increasing size of the raw graphite. This is because the specific surface area and charge transfer resistance simultaneously have a significant influence on the performances of the GO electrodes. At the same time, the three GO electrodes possess preeminent cycling stability.

As for the problem that appeared in the synthesis of GO, the decrease in specific surface area is from the contribution of hydrogen bonding interactions caused by intercalated water molecules. Further, small particles are more likely to agglomerate or even restack because of strong interactions between the GO layers. Thus, more attention should be paid to this problem in terms of synthesizing graphene oxide-based electrode materials for supercapacitors or other energy storage devices.

\section{Acknowledgements}

The authors acknowledge the financial support of this work by the National Natural Science Foundation of China (51302219, 51472204 and 51402236), the Natural Science Foundation of Shaanxi Province (2015JM2045), the Research Fund of the State Key Laboratory of Solidification Processing (NWPU), China (Grant No. 06-QP-2014), and the Fundamental Research Funds for the Central Universities (3102014JCQ01019 and 3102015BJ(II)MYZ02).

\section{Notes and references}

1 C. Liu, Z. Yu, D. Neff, A. Zhamu and B. Z. Jang, Nano Lett., 2010, 10, 4863-4868.

2 Y. Wang, Z. Shi, Y. Huang, Y. Ma, C. Wang, M. Chen and Y. Chen, J. Phys. Chem. C, 2009, 113, 13103-13107.

3 Y. A. Kim, T. Hayashi, J. H. Kim and M. Endo, J. Energy Chem., 2013, 22, 183-194.

4 M. D. Stoller, S. Park, Y. Zhu, J. An and R. S. Ruoff, Nano Lett., 2008, 8, 3498-3502.
5 Q. Zhang, K. Scrafford, M. Li, Z. Cao, Z. Xia, P. M. Ajayan and B. Wei, Nano Lett., 2014, 14, 1938-1943.

6 Y. Huang, J. Liang and Y. Chen, Small, 2012, 8, 1805-1834.

7 E. B. Secor, P. L. Prabhumirashi, K. Puntambekar, M. L. Geier and M. C. Hersam, J. Phys. Chem. Lett., 2013, 4, 1347-1351.

8 T. Yumura and A. Yamasaki, Phys. Chem. Chem. Phys., 2014, 16, 9656-9666.

9 X. Wang and G. Shi, Energy Environ. Sci., 2015, 8, 790-823.

10 B. Lobato, R. Wendelbo, V. Barranco and T. A. Centeno, Electrochim. Acta, 2014, 149, 245-251.

11 C. Soldano, A. Mahmood and E. Dujardin, Carbon, 2010, 48, 2127-2150.

12 C. H. Lui, L. Liu, K. F. Mak, G. W. Flynn and T. F. Heinz, Nature, 2009, 462, 339-341.

13 P. W. Sutter, J. I. Flege and E. A. Sutter, Nat. Mater., 2008, 7, 406-411.

14 F. Su, C. K. Poh, J. S. Chen, G. Xu, D. Wang, Q. Li, J. Lin and X. W. Lou, Energy Environ. Sci., 2011, 4, 717-724.

15 B. Xu, S. Yue, Z. Sui, X. Zhang, S. Hou, G. Cao and Y. Yang, Energy Environ. Sci., 2011, 4, 2826.

16 W. S. Hummers Jr and R. E. Offema, J. Am. Chem. Soc., 1958, 1339.

17 X. Zhang, K. Li, H. Li and J. Lu, J. Colloid Interface Sci., 2013, 409, 1-7.

18 A. C. Ferrari, Solid State Commun., 2007, 143, 47-57.

19 L. M. Malard, M. A. Pimenta, G. Dresselhaus and M. S. Dresselhaus, Phys. Rep., 2009, 473, 51-87.

20 Z. Ni, Y. Wang, T. Yu and Z. Shen, Nano Res., 2010, 1, 273291.

21 Z. H. Ni, T. Yu, Y. H. Lu, Y. Y. Wang, Y. P. Feng and Z. X. Shen, ACS Nano, 2008, 2301-2305.

22 S. Park and R. S. Ruoff, Nat. Nanotechnol., 2009, 4, 217-224.

23 D. R. Dreyer, S. Park, C. W. Bielawski and R. S. Ruoff, Chem. Soc. Rev., 2009, 39, 228-240.

24 Y. Yoon, K. Lee, C. Baik, H. Yoo, M. Min, Y. Park, S. M. Lee and H. Lee, Adv. Mater., 2013, 25, 4437-4444.

25 M. Acik, C. Mattevi, C. Gong, G. Lee, K. Cho, M. Chhowalla and Y. J. Chabal, ACS Nano, 2010, 4, 5861-5868.

26 J. H. Lee, N. Park, B. G. Kim, D. S. Jung, K. Im, J. Hur and J. W. Choi, ACS Nano, 2013, 7, 9366-9374.

27 A. Dimiev and J. M. Tour, ACS Nano, 2014, 8, 3060-3068.

28 M. Kruk and M. Jaroniec, Chem. Mater., 2000, 12, 1961-1968. 29 M. Kruk and M. Jaroniec, Chem. Mater., 2001, 13, 3169-3183. 30 R. Ryoo, I.-S. Park, S. Jun, C. W. Lee, M. Kruk and M. Jaroniec, J. Am. Chem. Soc., 2001, 123, 1650-1657.

31 Y. Li, M. Zhou, X. Cui, Y. Yang, P. Xiao, L. Cao and Y. Zhang, Electrochim. Acta, 2015, 161, 137-143.

32 R. N. Reddy and R. G. Reddy, J. Power Sources, 2006, 156, 700-704. 